\title{
Study on the Identification and Restricted Collocation of English and Chinese Phrase Structure
}

\author{
Shaoxia Zhan \\ School of Foreign Languages \\ Xuzhou Institute of Technology \\ Xuzhou, China 221008
}

\begin{abstract}
As for the definition of phrase structure, in a broad sense, it is a kind of language unit which does not include recursive structure. In the narrow sense, it is a structurally specific and stable vocabulary collocation form in the language which exists as dictionary knowledge, mainly including idioms and phrases. Restricted collocation is an important part of the language. Essentially, it is a kind of language phenomenon, and it usually has a normal and logical form of collocation, but there are also illogical and irregular collocation forms. The restricted collocation of English and Chinese phrase structure has a nature of polysemy. For this reason, it is necessary to strengthen the study of the restricted collocation of English and Chinese phrases. In this paper, the author analyzes the identification of English and Chinese phrase structure, and discusses the restricted collocation of English and Chinese phrase structure for reference.
\end{abstract}

Keywords-English and Chinese phrase structure; identification; restricted collocation

\section{INTRODUCTION}

In natural language, phrase is a syntactic element between sentences and vocabularies. In the process of foreign language teaching, the identification of English and Chinese phrase structure is a very important part. Restricted collocation is an important component which shall not be ignored in the language. In recent years, it attracts more and more attention from the research scholars. But at the present stage, the studies on the identification of English and Chinese phrase structure and restricted collocation are relatively less. For this reason, the author analyzes the identification of English and Chinese phrase structure, and studies the restricted collocation of English and Chinese phrase structure, hoping to make up for this gap and expecting to make a certain contribution for promoting further development and improvement of foreign language teaching in higher education in China.

\section{IDENTIFICATION OF ENGLISH AND CHINESE PHRASES}

For most phrases, the main basis of its internal structure is the characteristic syntactic structure of language. However, in the course of long-term use, some transferred meaning and extended meaning will inevitably appear, and such condition

Project: Foreign language subject of excellent project of social science application research Jiangsu Province 2015 (Contrastive Study on Automatic Identification and Restricted Collocation of English and Chinese Phrases)

Project Number: 15jsyw-37 may lead to great difference between the literal meaning and actual meaning of phrases. For example, the phrase "The Milky Way", literally, refers to the "milk path" from the literal sense; but from the actual sense, it refers to the "galaxy". It can be seen that in terms of meaning expression, the phrase is of atomicity, and its meaning cannot be obtained by combining the meaning of all the individuals that form the phrase. For this reason, the combination of basic individual vocabulary in machine translation is not tenable from the perspective of phrase translation, and phrase structure requires a separate identification method [1]. In addition, the framework of phrase structure has become one of the main means to eliminate ambiguity in natural language identification as it is relatively fixed. For some phrases, especially the verb phrases, compared to its core vocabulary, there is great difference in the syntactic function and syntactic features of the phrase. Based on this, the overall analysis of the phrase will not only provide a new idea for syntactic analysis, but also can reduce the difficulty of meaning conversion in English-Chinese translation process.

The most widely used English and Chinese phrase identification method is the maximum matching method based on the dictionary, whose basic idea is similar to the Chinese segmentation algorithm established based on positive maximum match, but this method can only be used to identify the phrases that are the same with those stored in the dictionary, and cannot identify the phrases with inflection and Verb-noun framework. The development and application of computeraided reading system, ectran English-Chinese translation system can effectively help to identify various phrase structures with complex phrasal structure. The principle is to apply the CERDL rules description language of LSD method to realize the description of individual vocabulary rules.

\section{INTRODUCTION TO RESTRICTED COLLOCATION}

According to different strength of combination ability, the combination of words can be divided into three types, namely, fixed collocation, restricted collocation and free collocation. Phrase structure usually involves different ranges; therefore, there are also certain differences in its definition. The different definitions indicate that there is certain difference in the research object and the scope of study. Because there is great difference in terminology heritage, research tradition and so on, "restricted collocation", in fact, refers to the so called "locution phrase" in academic community. In essence, locution phrase 
belongs to the category of idioms, referring to fixed phrases or commonly used fixed phrases. Normally, locution phrases will be regarded as a complete unit for practical use, and the composition of locution phrases cannot be changed arbitrarily. Locution phrases are the same as other idioms, having two characteristics of integral meaning and stable structure.

\section{IDENTIFICATION OF RESTRICTED COLLOCATION SYNTACTIC STRUCTURE}

The syntactic structure of restricted collocation is diverse, mainly including the verb-complement structure, parallel structure, modifier-head construction, verb-object construction and so on. Among them, modifier-head construction and verbobject construction are the two most common syntactic structures. For this reason, the industry mainly focuses on the modifier-head construction and verb-object construction in terms of study on restricted collocation [2]. For the internal relationship of the restricted collocation, it can be divided into two types according to its internal composition structure, that is, dominance relation and modifying relation; from the composition form of collocation, there are not only the normal and logical collocation forms, but also the non-logical and irregular collocation forms.

First is the dominance relation. The restricted collocation with this relation is usually reflected as verb-object construction. The more commonly used phrases include "act the goat", "fly the flag", "pour cold water", "cover your back" and so on. For the verb-object construction, as the name implies, its restricted collocation usually consists of two parts, the noun and the verb, that serve as object. For example, "cover your back" is mainly composed of "your back" that serves as object and the verb "cover", the former serves as the governed and the latter serves as the dominator of action. In the literal sense, the meaning of this phrase is "to cover your back", but the combination of literal meaning does not represent its true meaning; from the actual meaning point of view, it means "to prepare for the criticism to be suffered in the future and the supply". It can be seen that it is of the feature of integrity. In the Chinese language, there are also some "locution phrases" with dominance relation, generally it presents as verb-object construction, such as "give the green light", "beat about the bush" [3]. Compared to the English phrases, these "locution phrases" are also composed of nouns and verbs who serve as the object, such as "give a green light", which is mainly composed of the noun "green light" that serves as object and the verb "give", the former is the governed and the latter is the dominator of action; from the literal sense, "green light" refers to the green light in the traffic lights, "give a green light" means to open the green one of the traffic lights. However, there is no the real object of "green light", nor the action "turn on the light"; from the perspective of actual meaning, "give a green light" refers to "provide convenience, allow to do something."

Second is the modifying relation. The restricted collocation with such relation generally presents as modifier-head construction, and the more commonly used phrases include "the cat's whiskers", "the evil moment" and so on. Usually, such restricted collocation is used to one part to decorate another part of it. For example, "the cat's whiskers" is mainly composed of the noun "whiskers" and the possessive case of noun "cat's", the former is the modified, and the latter is the modifier. Literally, "whiskers" refers to beard, and "cat's" refers to something of the cat, so the meaning of this phrase is the cat's beard. However, the combination of literal meaning does not represent its true meaning; from the perspective of actual meaning, it refers to the "best people, ideas or things", which is of the feature of integrity. This restricted collocation has certain meaning of sarcasm, because there is nothing good of cat's beard, so that people will have the association of "someone who does not have ability but believes he is great" [4]. In addition, part of the restricted collocation with modifying relation can be replaced under normal conditions, without meaning being changed, for example, "whiskers" in the cat's whiskers can be replaced by "pyjamas". In Chinese, there are also some "locution phrases" with modifying relation. Under normal circumstances, they are represented as modifierhead construction; the more commonly used ones include "cold bench", "mind relief" and so on. Normally, such restricted collocation is to use one part to decorate another part of it. Such as "cold bench" mainly formed by the noun "bench" and adjective "cold"; the former is the modified, and the latter is the modifier. In the literal sense, the "bench" refers to the stool made of wood, the adjective "cold" refers to the lower temperature; as a result, from the literal point of view, "cold bench" refers to the stool made of wood in the lower temperature [5]. However, the "cold bench" as a "locution phrase", its true meaning cannot be expressed by combining various words in this phase, as it has the feature of integrity, which is a very vivid metaphor, and it is easy to make people think of the meaning of "unpopular, being treated coldly". In addition, one part of the "locution phrase" with modifying relation can usually be replaced; for example, the adjective "cold" in "cold bench" can be replaced by another adjective "cool", without meaning being changed.

From the perspective of integration degree, there is certain difference in the restricted collocation and "locution phrase" of verb-object construction and that of modifier-head construction. From the above analysis it can be seen that, the restricted collocation and "locution phrase" of modifier-head construction has a quite close integration degree, with no other components can be added. Moreover, in the actual use process, usually no variant in terms of forms will appear, and EnglishChinese restricted collocation has the same expression form in this respect.

\section{FUNCTIONAL CLASSIFICATION OF RESTRICTED COLLOCATION}

Restricted collocation mainly has three functions, namely, the semantic meaning, emotional function and coherent function. First is the semantic function. In the language system, the restricted collocation is an important part that cannot be ignored. Moreover, in the actual communication process, the restricted collocation has also been widely used by people. On the whole, the restricted collocation has a conceptual meaning, and it can also express the meaning of an object via the concept meaning, indicating that the restricted collocation has the semantic function; the second is emotional function. Restricted collocation is of a very intense spoken language feature, which 
can fully express the mental association, colloquial style and expression of the language unit. Compared to the ordinary words, restricted collocation is more vivid, and has different meanings, such as neutral meaning, derogatory sense and commendatory sense, etc., which can fully display people's emotions, reveal that restricted collocation is of emotional function [7]; third is coherent function. For the restricted collocation, it can link the meaning of the whole text organically through the relationship of interpersonal meaning and conceptual meaning, so as to achieve the completeness and coherence of the whole text, which indicates that restricted collocation has the coherent function. However, considering from a practical perspective, coherence also belongs to the scope of psychological performance. In addition, language has a certain degree of particularity, as it not only has the psychological logic, but also has a strong social interaction function and communicative functions, being able to achieve socialization of language psychological content as well as to express emotions, attitudes and social relations. In society and the process of thinking, the language has played a role of bridge and link. In addition, society and thinking also has a mutual interaction each other, which is mainly reflected in the following relations, namely, the society can create thinking, and thinking in turn can promote the society, which indicates that thinking, language and society are inseparable from each other.

\section{CONCLUSION}

In summary, the identification of English and Chinese phrase structure and restricted collocation have attracted more and attention in recent years. It is of great significance to foreign language teaching, English-Chinese translation and dictionary compilation to strengthen the study of English and Chinese phrase structure collating collocation. Based on the English and Chinese languages, this paper compares and analyzes the identification of the restricted collocation syntactic structure and discusses the functions of restricted collocation, and it is found that the restricted collocation has semantic function, emotional function and coherence function.

\section{REFERENCES}

[1] Huang Xiaoling. Study on Correction Strategies of College English Composition based on Ripple Effect of Restricted Collocation [J]. Journal of Hubei University of Economics (Humanity and Social Science Edition), 2016,(11):213-214.

[2] Liu Lijin, Deng Weiping. Analysis of Topological Relationship Expression and Its Reasons [J]. Journal of Beijing International Studies University, 2016,(02):1-14+131.

[3] Zhen Fengchao. Exploration of English-Chinese Translation Unit from Valency Structure - based on the Study of Corpus [J]. Foreign Language Teaching and Research, 2016,(03):442-454+480.

[4] Yang Jingpeng, Wu Hongyun. Syntax of English and Chinese Spatial Boundary in Sports Events Framework-Empirical Study on Semantic Interface_taking "walk through" as an Example [J]. Foreign Language Teaching, 2015,(04):33-36.

[5] Zhou Jun. Analysis of Syntactic Hierarchy of English - Chinese Noun Phrases [J]. Foreign Languages (Journal of Shanghai International Studies University), 2014, (05):18-27.
[6] He Xiaowei, Zhong Lanmei. Study on the Formation of English Chinese Middle Construction under the Minimalist Program [J]. Modern Foreign Languages, 2012,(01):14-22+108.

[7] Ma Jianjun, Huang Degen. Research on English Functional Noun Phrases and Its Application [J]. Journal of Dalian University of Technology, 2012,(01):126-131. 